\title{
Temperature Dependence of the Energy Band Diagram of AlGaN/GaN Heterostructure
}

\author{
Yanli Liu (D), ${ }^{1}$ Dunjun Chen $\left(\mathbb{D},{ }^{2}\right.$ Kexiu Dong, ${ }^{3}$ Hai Lu, ${ }^{2}$ Rong Zhang, \\ Youdou Zheng, ${ }^{2}$ Zhilin Zhu, ${ }^{1}$ Guangfen Wei, ${ }^{1}$ and Zhonghai Lin ${ }^{1}$ \\ ${ }^{1}$ Key Laboratory of Intelligent Information Processing in Universities of Shandong, \\ School of Information and Electronic Engineering, Shandong Technology and Business University, Yantai 264005, China \\ ${ }^{2}$ Key Laboratory of Advanced Photonic and Electronic Materials, School of Electronic Science and Engineering, \\ Nanjing University, Nanjing 210093, China \\ ${ }^{3}$ School of Electronic and Electrical Engineering, Chuzhou University, Chuzhou 239000, China
}

Correspondence should be addressed to Yanli Liu; yanliliu0727@163.com and Dunjun Chen; djchen@nju.edu.cn

Received 5 January 2018; Accepted 30 January 2018; Published 1 April 2018

Academic Editor: Shenghuang Lin

Copyright (c) 2018 Yanli Liu et al. This is an open access article distributed under the Creative Commons Attribution License, which permits unrestricted use, distribution, and reproduction in any medium, provided the original work is properly cited.

\begin{abstract}
Temperature dependence of the energy band diagram of AlGaN/GaN heterostructure was investigated by theoretical calculation and experiment. Through solving Schrodinger and Poisson equations self-consistently by using the Silvaco Atlas software, the energy band diagram with varying temperature was calculated. The results indicate that the conduction band offset of $\mathrm{AlGaN} / \mathrm{GaN}$ heterostructure decreases with increasing temperature in the range of $7 \mathrm{~K}$ to $200 \mathrm{~K}$, which means that the depth of quantum well at $\mathrm{AlGaN} / \mathrm{GaN}$ interface becomes shallower and the confinement of that on two-dimensional electron gas reduces. The theoretical calculation results are verified by the investigation of temperature dependent photoluminescence of AlGaN/GaN heterostructure. This work provides important theoretical and experimental basis for the performance degradation of AlGaN/GaN HEMT with increasing temperature.
\end{abstract}

\section{Introduction}

AlGaN/GaN high electron mobility transistor (HEMT) has attracted great interest for high temperature, high frequency, and high power applications due to its intrinsic material advantages, such as wide band gap, high breakdown electric field, high electron saturation velocity, and high two-dimensional electron gas (2DEG) concentration [1-3]. Although the performance of AlGaN/GaN HEMT has made remarkable progress [4-7], the reliability, especially at high temperature, has been and remains a major constraint in realizing the true potentials of such devices [8-10]. Improvements of reliability require a better understanding of the degradation mechanism. The performance degradation of AlGaN/GaN HEMTs with increasing temperature can be largely attributed to the effect of temperature on 2 DEG transport properties [10-12]. The intrinsic physical reason for that is the energy band diagram of $\mathrm{AlGaN} / \mathrm{GaN}$ heterostructure varies with temperature, which should be fully investigated.
However, there have been few reports on the temperature dependence of the energy band structure of AlGaN/GaN heterostructures until now. Wang et al. [13] calculated the energy band diagram of $\mathrm{AlGaN} / \mathrm{GaN}$ heterostructure at room temperature, $250^{\circ} \mathrm{C}$ and $500^{\circ} \mathrm{C}$, just to explain that the $2 \mathrm{DEG}$ density decreases with increasing temperature. But there is not detailed analysis and discussion. In this work, the temperature dependence of the energy band structure of $\mathrm{AlGaN} / \mathrm{GaN}$ heterostructure was investigated by theoretical calculation and experimental verification.

\section{Theoretical Calculation}

The AlGaN/GaN heterostructure used in this study consists of a $2 \mu \mathrm{m}$ thick GaN buffer layer and a $25 \mathrm{~nm}$ thick AlGaN barrier layer, as shown in Figure 1. The Al composition of the AlGaN layer is 0.3 . The $n$-type doping level in both GaN and AlGaN layers is set to be $1 \times 10^{16} \mathrm{~cm}^{-3}$, to keep consistent with the level for unintentionally doped samples 


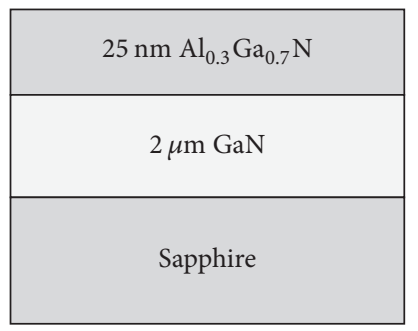

FIGURE 1: Schematic of AlGaN/GaN heterostructure.

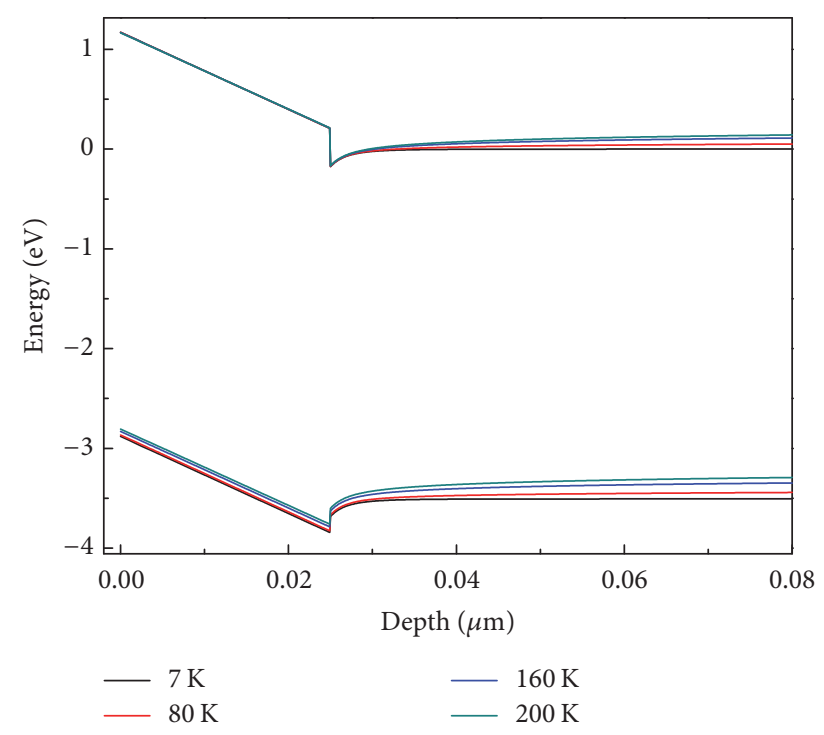

FIGURE 2: The energy band diagrams of AlGaN/GaN heterostructure at different temperature.

in experiment. Considering the screening effect caused by defects, the polarization charge densities are assumed to be $40 \%$ of the calculated values [14].

Through solving the Schrodinger and Poisson equations self-consistently by using the Silvaco Atlas software [15], the temperature dependent energy band diagram of $\mathrm{AlGaN} / \mathrm{GaN}$ heterostructure can be calculated. The calculation results with temperature lower than $200 \mathrm{~K}$ are shown in Figure 2. It can be seen that the conduction and valence band energies of both $\mathrm{GaN}$ and AlGaN layers increase with increasing temperature, especially in the GaN layer. Compared with the conduction band, the valence band shows larger shift with varying temperature. So, the energy band gaps of GaN and AlGaN layers decrease with increasing temperature. This is consistent with temperature dependent band gap shrinkage effect $[16,17]$.

Besides the energy band gaps, the conduction band offset of $\mathrm{AlGaN} / \mathrm{GaN}$ heterostructure also changes with temperature. The conduction band profile near the AlGaN/GaN interface with varying temperature is shown in Figure 3. It can be seen that the conduction band offset between AlGaN and $\mathrm{GaN}$ layers decreases with increasing temperature, which means that the depth of the quantum well at $\mathrm{AlGaN} / \mathrm{GaN}$ interface becomes shallower and the confinement of that

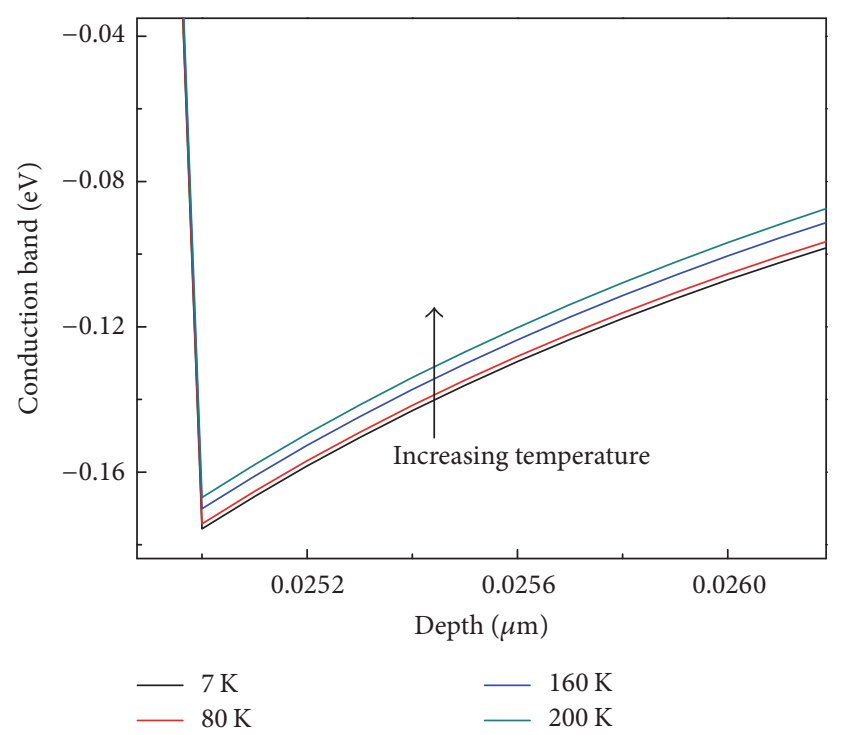

FIGURE 3: The conduction band profile of the AlGaN/GaN interface for different temperature.

on $2 \mathrm{DEG}$ reduces. In addition, the $2 \mathrm{DEG}$ concentration in unintentionally doped $\mathrm{AlGaN} / \mathrm{GaN}$ heterostructures shows a direct proportional relationship to the conduction band offset, according to the following equation [13]:

$$
\begin{aligned}
n_{s}(x)= & \frac{\sigma(x)}{e} \\
& -\left(\frac{\varepsilon_{0} \varepsilon(x)}{d_{\mathrm{AlGaN}} e^{2}}\right)\left[e \phi_{b}(x)+E_{F}(x)-\Delta E_{c}(x)\right],
\end{aligned}
$$

where $\sigma(x)$ is the polarization-induced bound charge, $\varepsilon(x)$ is the relative dielectric constant of $\mathrm{AlGaN}, d_{\mathrm{AlGaN}}$ is the thickness of the $\mathrm{Al}_{x} \mathrm{Ga}_{1-x} \mathrm{~N}$ barrier layer, $e \phi_{b}(x)$ is the Schottky barrier of the gate contact on top of $\mathrm{AlGaN}, E_{F}(x)$ is the Fermi level with respect to the GaN conduction-bandedge energy, and $\Delta E_{c}(x)$ is the conduction band offset at AlGaN/GaN interface. So, the 2DEG concentration decreases with increasing temperature due to the reduction of the conduction band offset $\Delta E_{c}$. The same temperature dependence of the 2DEG concentration has been measured by Khan et al. [18]. Therefore, the performance of AlGaN/GaN HEMT will degrade with increasing temperature [19]. The detailed temperature dependence of the energy band gaps and conduction band offset in $\mathrm{AlGaN} / \mathrm{GaN}$ heterostructure is shown in Figure 4.

\section{Experimental Verification}

In order to verify the theoretical calculation, we investigated temperature dependent photoluminescence (PL) of $\mathrm{AlGaN} / \mathrm{GaN}$ heterostructure, which can directly reflect the energy band structure of the measured samples. In this work, PL measurements were performed on the $\mathrm{Al}_{0.3} \mathrm{Ga}_{0.7} \mathrm{~N} / \mathrm{GaN}$ heterostructure between $7 \mathrm{~K}$ and $200 \mathrm{~K}$. The light source was a He-Cd laser with a wavelength of $325 \mathrm{~nm}$. 


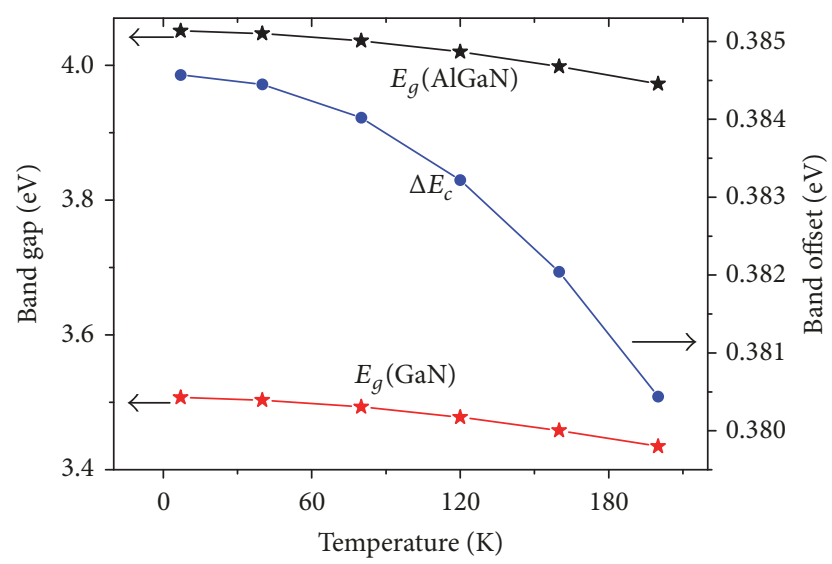

FIgURE 4: Temperature dependence of the energy band gaps of $\mathrm{AlGaN}$ and $\mathrm{GaN}$ and the conduction band offset of AlGaN/GaN heterostructure.

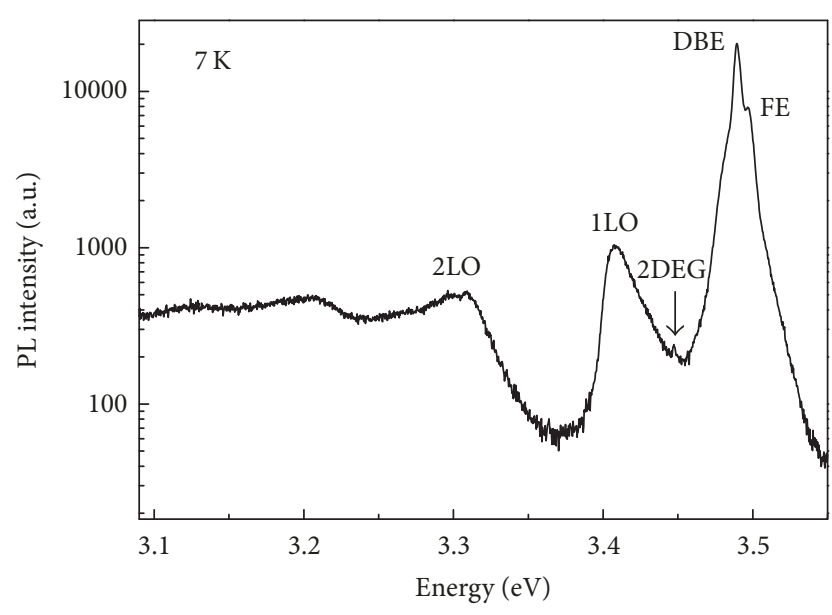

FIGURE 5: PL spectrum of AlGaN/GaN heterostructure at $7 \mathrm{~K}$.

Figure 5 shows the PL spectrum of the AlGaN/GaN heterostructure at $7 \mathrm{~K}$. The free exciton $(\mathrm{FE})$ and donor bound exciton (DBE) emissions in $\mathrm{GaN}$ are located at $3.498 \mathrm{eV}$ and $3.489 \mathrm{eV}$, respectively, and are much stronger than other peaks. These two emissions are near-band-edge emissions, which can directly reflect the band gap of corresponding material. The broad peaks at $3.408 \mathrm{eV}$ and $3.309 \mathrm{eV}$ are attributed to the one and two longitudinal optical (LO) phonon replicas of the GaN FE emission, respectively. The weak peak at $3.448 \mathrm{eV}$ is attributed to recombination between 2DEG and photoexcited holes. Due to the strong built-in internal electric field near AlGaN/GaN heterointerface, the photoexcited holes diffuse rapidly into the flat-band region of GaN. Therefore, the probability of recombination between 2DEG and photoexcited holes is low and its intensity is very weak.

Figure 6 shows the PL spectra of AlGaN/GaN heterostructure with varying temperature in the range of $7 \mathrm{~K}$ to $160 \mathrm{~K}$. The inset shows the PL spectrum at $200 \mathrm{~K}$. It can be seen from Figure 6 that the GaN FE and DBE emissions

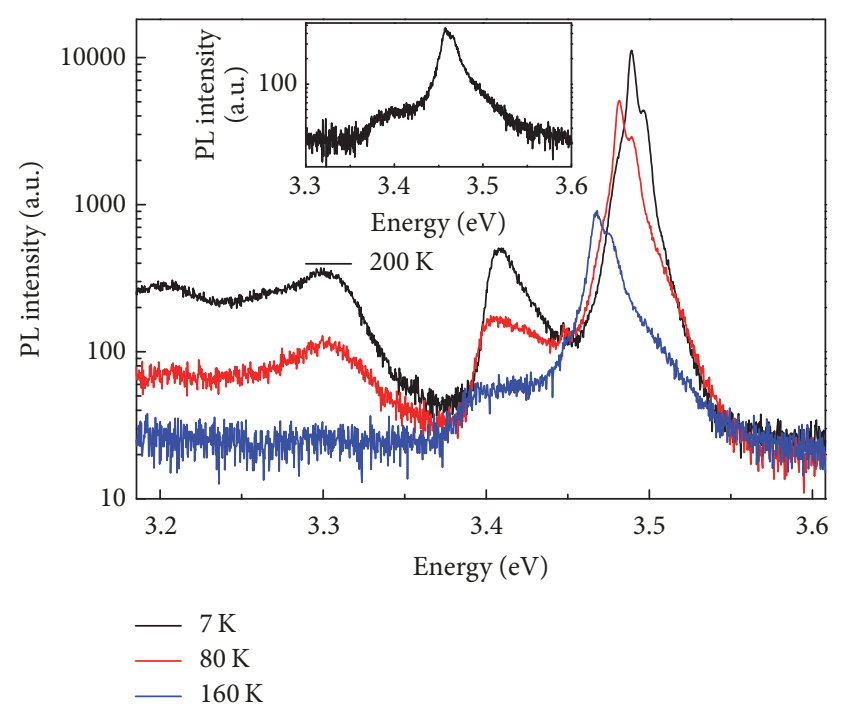

Figure 6: PL spectra of AlGaN/GaN heterostructure in the temperature range of $7 \mathrm{~K}$ to $160 \mathrm{~K}$. The inset shows the PL spectrum of $\mathrm{AlGaN} / \mathrm{GaN}$ heterostructure at $200 \mathrm{~K}$.

exhibit obvious red shift with increasing temperature, indicating the reduction of the band gap of GaN layer. This is consistent with the result of theoretical calculation. The intensity of the 2DEG PL peak is very weak and decreases with increasing temperature. As shown in the inset of Figure 6 , this peak disappears when the temperature reaches $200 \mathrm{~K}$. Different from the GaN FE and DBE peaks, the 2DEG PL peak shows unobvious shift with increasing temperature. It demonstrates that the energy separation between the ground state of 2DEG and the valence band of flat-band region in GaN layer does not change obviously with varying temperature in the range of $7 \mathrm{~K}$ to $200 \mathrm{~K}$. Additionally, the energy separation between GaN FE and 2DEG PL peak gradually decreases with increasing temperature, which indicates the depth of quantum well at AlGaN/GaN interface becomes shallower. Therefore, the confinement of the interface quantum well on 2DEG decreases with increasing temperature. It is also consistent with the theoretical calculation results.

\section{Conclusions}

In summary, temperature dependence of the energy band diagram of $\mathrm{AlGaN} / \mathrm{GaN}$ heterostructure was investigated. Through theoretical calculation and experiment verification, it is confirmed that the band gaps of both AlGaN and $\mathrm{GaN}$ layers and the conduction band offset of AlGaN/GaN heterostructure decrease with increasing temperature in the range of $7 \mathrm{~K}$ to $200 \mathrm{~K}$. So the depth of quantum well at $\mathrm{AlGaN} / \mathrm{GaN}$ interface becomes shallower and the confinement of that on $2 \mathrm{DEG}$ reduces. This work provides important theoretical and experimental basis for the performance degradation of AlGaN/GaN HEMT with increasing temperature. 


\section{Conflicts of Interest}

The authors declare that there are no conflicts of interest regarding the publication of this paper.

\section{Acknowledgments}

This work was supported by the National Natural Science Foundation of China (nos. 61634002, 61474060, and 61174007), National Key R\&D Program of China (2017YFB0402900), the Natural Science Foundation of Shandong Province (ZR2016FP09), the Project of Shandong Province Higher Educational Science and Technology Program (J16LN04), the Key Project of Jiangsu Province (BE2016174), the Yantai Key R\&D Program (nos. 2017ZH063, 2017ZH064, and 2016ZH053), the PhD Start-Up Fund of Shandong Technology and Business University (BS201608), and the Natural Science Foundation of Anhui Province (1708085MF149).

\section{References}

[1] U. K. Mishra, P. Parikh, and Y.-F. Wu, "AlGaN/GaN HEMTs-an overview of device operation and applications," Proceedings of the IEEE, vol. 90, no. 6, pp. 1022-1031, 2002.

[2] A. Eblabla, X. Li, I. Thayne, D. J. Wallis, I. Guiney, and K. Elgaid, "High performance GaN high electron mobility transistors on low resistivity silicon for X-band applications," IEEE Electron Device Letters, vol. 36, no. 9, pp. 899-901, 2015.

[3] Y. Tang, K. Shinohara, D. Regan et al., "Ultrahigh-speed GaN high-electron-mobility transistors with fTfmax of 454/444 GHz," IEEE Electron Device Letters, vol. 36, no. 6, pp. 549-551, 2015.

[4] L. F. Eastman, V. Tilak, V. Kaper et al., "Progress in high-power, high frequency AlGaN/GaN HEMTs," Physica Status Solidi (a) - Applications and Materials Science, vol. 194, no. 2, pp. 433-438, 2015.

[5] S. Huang, X. Liu, J. Zhang et al., "High RF Performance Enhancement-Mode Al2O3/AlGaN/GaN MIS-HEMTs Fabricated with High-Temperature Gate-Recess Technique," IEEE Electron Device Letters, vol. 36, no. 8, pp. 754-756, 2015.

[6] M. Zhang, X.-H. Ma, M.-H. Mi et al., "Improved on-state performance of AlGaN/GaN Fin-HEMTs by reducing the length of the nanochannel," Applied Physics Letters, vol. 110, no. 19, Article ID 193502, pp. 287-292, 2017.

[7] L. Shen, D. Zhang, X. Cheng et al., "Performance Improvement and Current Collapse Suppression of Al2O3/AlGaN/GaN HEMTs Achieved by Fluorinated Graphene Passivation," IEEE Electron Device Letters, vol. 38, no. 5, pp. 596-599, 2017.

[8] G. Meneghesso, G. Verzellesi, F. Danesin et al., "Reliability of GaN high-electron-mobility transistors: State of the art and perspectives," IEEE Transactions on Device and Materials Reliability, vol. 8, no. 2, pp. 332-343, 2008.

[9] Y. C. Chou, D. Leung, I. Smorchkova et al., "Degradation of AlGaN/GaN HEMTs under elevated temperature lifetesting," Microelectronics Reliability, vol. 44, no. 7, pp. 1033-1038, 2004.

[10] J. Yang, X. Zhang, and C. Lv, "Study on the reliability of AlGaN/ GaN HEMTs at high temperature," in Proceedings of the 2011 International Conference on Electric Information and Control Engineering (ICEICE '11), pp. 774-777, China, April 2011.
[11] A. M. Darwish, B. D. Huebschman, E. Viveiros, and H. A. Hung, "Dependence of GaN HEMT millimeter-wave performance on temperature," IEEE Transactions on Microwave Theory and Techniques, vol. 57, no. 12, pp. 3205-3211, 2009.

[12] G. J. Syaranamual, W. A. Sasangka, R. I. Made et al., "Role of two-dimensional electron gas (2DEG) in AlGaN/GaN high electron mobility transistor (HEMT) ON-state degradation," Microelectronics Reliability, vol. 64, pp. 589-593, 2016.

[13] M. J. Wang, B. Shen, F. J. Xu et al., "High temperature dependence of the density of two-dimensional electron gas in Al0.18Ga0.82N/GaN heterostructures," Applied Physics A: Materials Science \& Processing, vol. 88, no. 4, pp. 715-718, 2007.

[14] K. Dong, D. Chen, B. Liu et al., "Characteristics of polarizationdoped N-face III-nitride light-emitting diodes," Applied Physics Letters, vol. 100, no. 7, Article ID 073507, 2012.

[15] A. Abou-Elnour and K. Schuenemann, "A comparison between different numerical methods used to solve Poisson's and Schroedinger's equations in semiconductor heterostructures," Journal of Applied Physics, vol. 74, no. 5, pp. 3273-3276, 1993.

[16] N. Sarkar and S. Ghosh, "Temperature dependent band gap shrinkage in GaN: Role of electron-phonon interaction," Solid State Communications, vol. 149, no. 31-32, pp. 1288-1291, 2009.

[17] N. Nepal, J. Li, M. L. Nakarmi, J. Y. Lin, and H. X. Jiang, "Temperature and compositional dependence of the energy band gap of AlGaN alloys," Applied Physics Letters, vol. 87, no. 24, Article ID 242104, pp. 1-3, 2005.

[18] A. Khan, F. Shah, J. Siddiq, and T. Vasen, "High temperature transport properties of $\mathrm{AlGaN} / \mathrm{GaN}$ heterostructures," $N d . E d u$, vol. 1, pp. 1-4, 2010.

[19] B. A. Polash and H. Huq, "High temperature performance measurement and analysis of GaN HEMTs," in Proceedings of the Society of Photo-Optical Instrumentation Engineers (SPIE), vol. 6894, January 2008. 

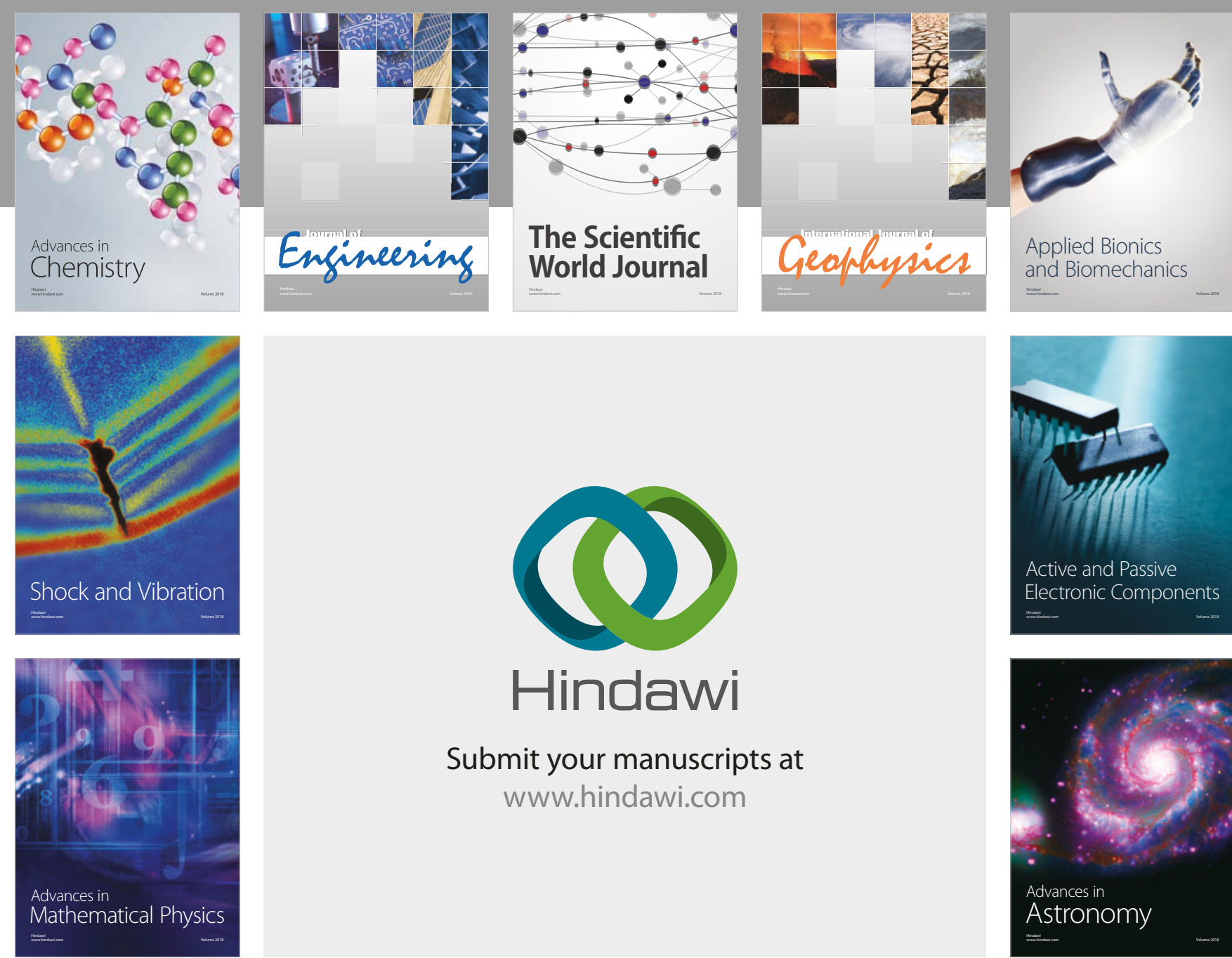

Submit your manuscripts at

www.hindawi.com

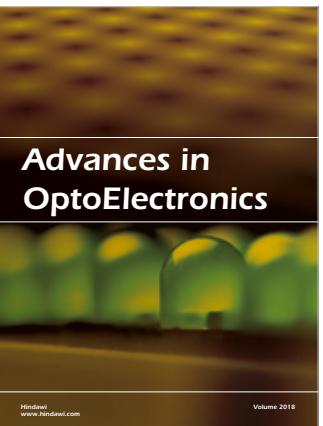

\section{Rotcting Machinery}
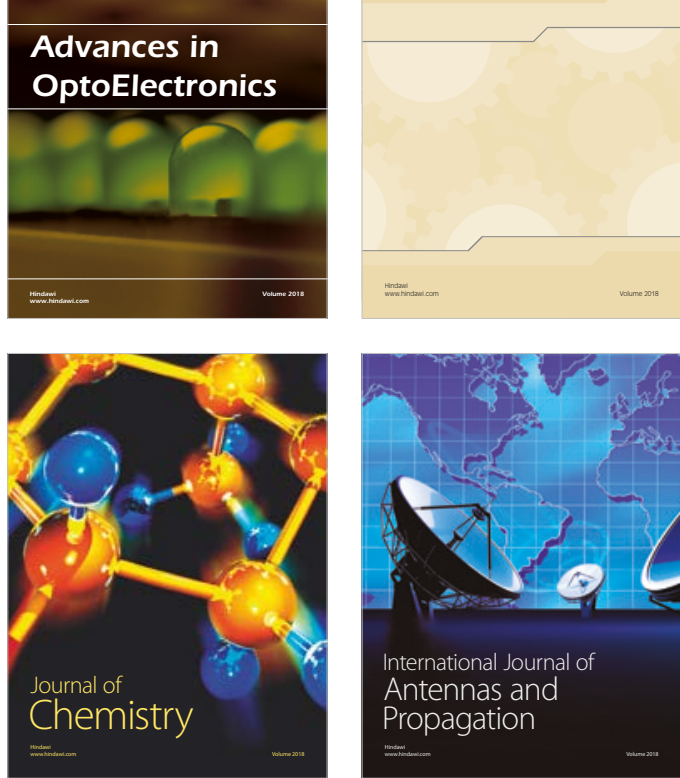

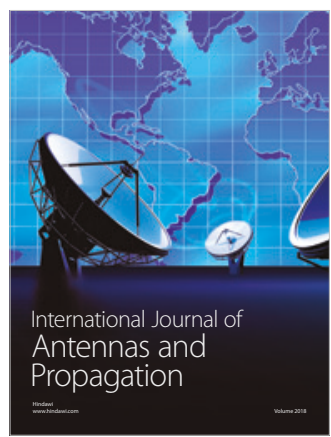

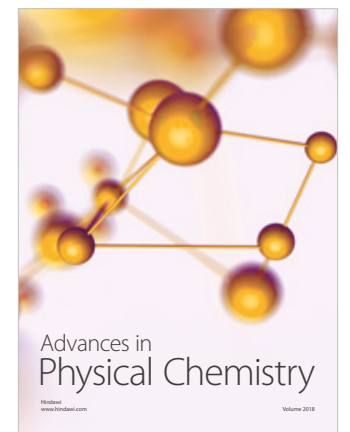

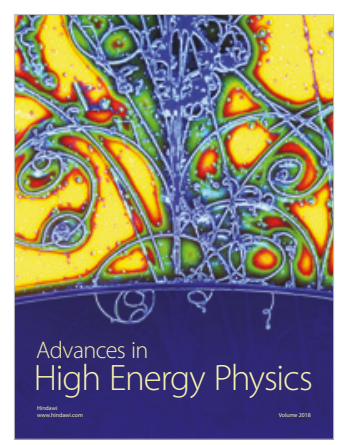

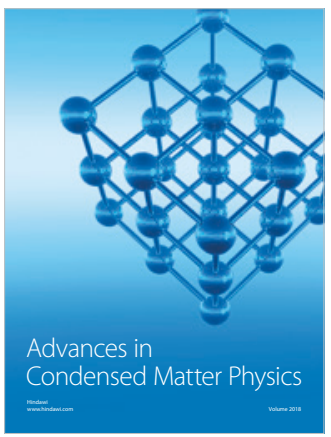

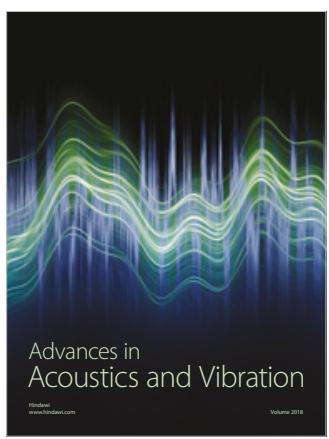

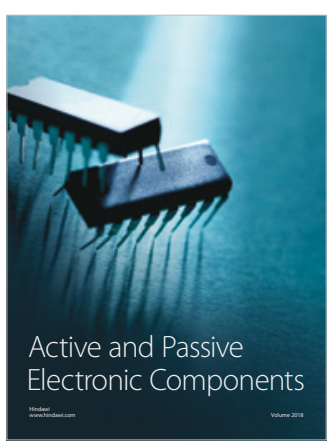
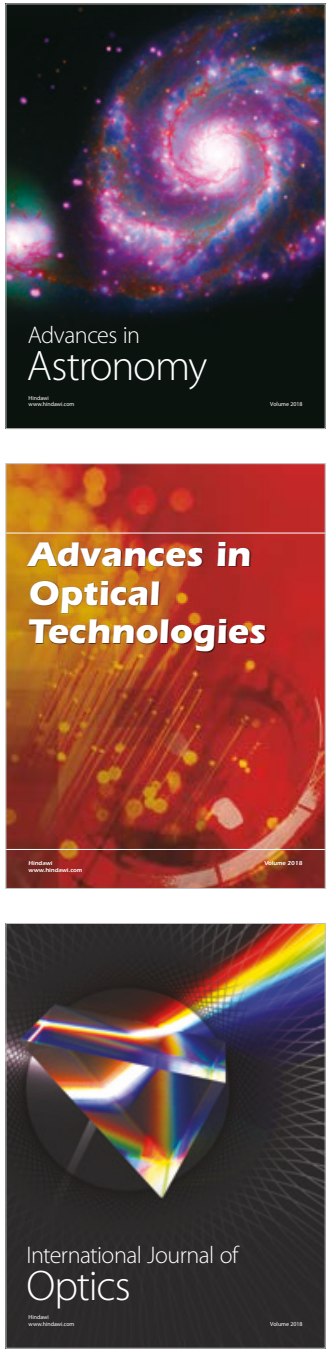